Research Article

\title{
Intelligent Network Office System Based on Cloud Computing and Machine Learning
}

\author{
Yinfang Guo' and Yanjun Guo ${ }^{1}{ }^{2}$ \\ ${ }^{1}$ Department of Computer Science and Technology, Taiyuan University, Taiyuan 030032, China \\ ${ }^{2}$ Department of Computer Engineering, Shanxi Engineering Vocational College, Taiyuan 030009, China \\ Correspondence should be addressed to Yanjun Guo; guoyanjun@st.btbu.edu.cn
}

Received 22 September 2021; Revised 16 October 2021; Accepted 10 November 2021; Published 10 December 2021

Academic Editor: Chin-Ling Chen

Copyright (c) 2021 Yinfang Guo and Yanjun Guo. This is an open access article distributed under the Creative Commons Attribution License, which permits unrestricted use, distribution, and reproduction in any medium, provided the original work is properly cited.

\begin{abstract}
The traditional data automatic office system has limited mining and computing capabilities. Due to the iterative complexity of data mining algorithms, it is difficult to discover the relationships and rules existing in the Internet of Things data as well as impossible to advance the efficiency of the office system based on the existing Internet of Things data. This paper combines cloud computing and machine learning to construct an intelligent network office system, realizes large-scale IoT data processing through the combination of IoT data mining technology and cloud computing framework, and constructs the functional module structure of the intelligent network office system through demand analysis. On this basis, this paper conducts system performance verification and conducts experimental design based on network intelligent system demand. The experimental results show that the system constructed in this paper has certain practical effects, which can provide theoretical reference for subsequent related research.
\end{abstract}

\section{Introduction}

In the early stage of the development of the information industry, information technology was not advanced enough. Software products were scarce resources, and the software market was a seller's market. Technical factors played a dominant role in software development. After successful software development, the corresponding market demand could be found. The key link of the informatization project at this stage is the writing of software code, and there is basically no need for targeted demand analysis. The success of software development indicates the success of the project. With the rapid development of information technology, the continuous expansion of the information industry, the shortening of the life cycle of information projects, and the introduction of personalized products, software systems have entered the buyer's market. Customers' requirements for software systems are constantly increasing, and their requirements for personalization, specificity, and customized software systems continue to increase. At this time, informatization projects enter the buyer's market, and customized software is the industry's development trend. Whether developers can develop products that meet customer needs and satisfy customers is the criterion for testing the success of software development projects [1].

At present, in the daily office management applications, there are a large number of process management and process processing issues, including resource coordination, task allocation, work scheduling, and control [2].

Due to insufficient process support capabilities, the existing office management system cannot truly realize the electronic management of official documents in the government. Electronic writing can replace manual writing, which solves the problems of slow transmission of official documents, untimely information, unsynchronized information, and difficult access and realizes official documents, electronic drafting, review, countersignature, issuance, registration, approval, circulation, and other tasks. The existing office management system cannot realize tasks such as automatic processing feedback, reminder, process tracking and statistics, query, and archiving of task personnel and business processes and cannot be flexible. Setting 
the official document process and conducting full-text search, the work quality and efficiency cannot be effectively tracked and implemented.

The main goal of the office automation system is to use a variety of information and communication technologies to realize the coordination and solidification of daily office management information resource sharing and management and office management processes, improve daily office management efficiency and daily office management quality, and assist office management decision-making [3].

This article introduces the background status and actual needs of intelligent network office, studies the current literature research status of cloud computing and intelligent network office, and analyzes the existing research problems. On this basis, this paper proposes a cloud computing application of intelligent clustering algorithm. With the support of the cloud computing clustering algorithm, an intelligent network office system is constructed, and the performance of the system is verified, which verifies the effectiveness of the system in this paper.

In terms of the depth of theoretical research in the above literature, the introduction of new technologies, and the promotion of mature products, especially in the application of workflow technology in office management systems, more domestic research is still theoretical, and there are relatively few practical application products. Workflow is the practical application of the office management system that needs further research. This article builds an intelligent network office system with the support of cloud computing technology and machine learning to improve the efficiency of intelligent office.

\section{Related Work}

Internet of Things was called the sensor network in the early days, and the sensor network technology was rated as the top ten science and technology that will change the future life of mankind [4]. In the era of Internet of Things, any object can be connected to each other through a communication network. However, Internet of Things has been a broad concept since it was proposed. Because it involves complex technologies such as information, wireless networks, and intelligent control, it is difficult to give it a clear and unified definition [5]. At present, the definition accepted by the mainstream is it refers to a network concept of the GPS, laser scanner, and other information-sensitive sensing devices that connect any item to the Internet according to an agreed protocol for information exchange and communication to realize intelligent identification, positioning, tracking, monitoring, and management [6]. At present, countries all over the world pay attention to the informatization development of science as well as technology. China, the United States, Japan, and South Korea have included Internet of Things in their national strategic plans [7]. At present, many experts and scholars are committed to the core technology research and application system development of Internet of Things so as to establish a technical standard system supported by independent intellectual property rights [8]. Cloud computing is a new computing method based on the
Internet, which provides users with on-demand computing through heterogeneous and autonomous services on the Internet [9]. Cloud computing has created countless new ways of working and business models. The Internet of Things realizes the interconnection and intercommunication of data and information between things, and things as well as the Internet, and cloud computing uses its powerful computing and storage capabilities to mine the knowledge laws contained in massive data [10]. The collaborative application of the Internet of Things and cloud computing is conducive to the comprehensive collection and intelligent processing of time data and improves the level of information management in the field [11].

Smart office, also called cloud office, is a new office model that uses cloud computing technology to intelligently manage software and hardware equipment required for office business and realizes the unified deployment and delivery of enterprise application software [12]. Smart office supports the secure remote access of multiple terminal devices such as PCs, mobile phones, and tablet computers, which can enhance the security, ease of use, and scalability of the corporate office environment, improve collaboration and sharing of resources, and comprehensively improve management efficiency and optimization business process, reducing operating costs [13]. Smart office has a wide range of development space and application prospects in the future. Smart office systems make up for most of the shortcomings of traditional office systems and bring office automation to a new level [14]. Daily office applications required by enterprises into virtual desktops, unified delivery, and management are integrated. No matter when and where, as long as users log in with their own account, they will get their own customized operating environment [15]. Cloud technology is used to build a new office application service. Users are provided with an operating experience of "desktop as a service, ready to use" [16]. It is proposed that the various functions provided by the smart office system can not only effectively improve the internal communication management process of the enterprise but also solve the common problems in the enterprise informatization process [17]. With the help of the smart office system, the unified management, rapid deployment, and flexible expansion of each system can be realized, which can effectively reduce the maintenance and management costs [18]. It is proposed that smart office meets the needs of new office applications such as mobile office. At the same time, the smart office system has advantages in reducing enterprise management costs, improving management level and efficiency, etc. [19]. It is proposed that smart office can reduce the IT cost caused by PC updates and can provide users with flexible configuration that cannot be achieved by ordinary PCs. The parameters of smart office desktops support independent setting of high-end or low-end configurations and are equipped on demand [20]. The centralized management of enterprise IT is realized through smart office. Centralized management and online support tools make enterprises no longer need to 
deploy IT staff in new locations. This can reduce costs, and enterprises can achieve easy, fast, and flexible development. This can reduce the cost of enterprises and accelerate the business process, and enterprises can achieve easy, rapid, and flexible development [21]. It is proposed that smart office can improve the security of enterprise data assets. All data are unified to run on the server side, and the client side only displays its changed images, and there is no need to worry about illegally stealing data through the client side [22]. It is proposed that smart office can realize flexible and convenient mobile office, create a new office model, and realize mobile office through cloud computing technology, and office staff can handle anything related to business at any time and any place. The flexible and intelligent mobile office will become a powerful driving force for companies to gain a competitive advantage; they can work efficiently with other employees in multiple different locations, no matter where they are, and transfer the low-value tasks of highskilled employees to low-cost employees to complete, which not only can reduce costs but also improve efficiency [23].

From the high analysis, we can see that the depth of theoretical research in the above literature, the introduction of new technologies, and the promotion of mature products, especially in the application of workflow technology in office management systems, are still more theoretical research in China, and there are relatively few practical application products. The practical application of workflow in the office management system needs further study.

\section{Cloud Computing Smart Office Data Clustering Processing}

As a new generation of data processing and storage technology, cloud computing has realized rapid data processing and mobile applications. As the problem of in-depth mining of massive data information has become an urgent problem to be solved, a clustering algorithm based on cloud computing is designed and applied to the massive data processing of the intelligent office to improve the operating efficiency of the intelligent office system. Clustering refers to reusing as much as possible the manual identification work done by the predecessors to improve work efficiency. It is not difficult to solve the formal data mining clustering problem. The easiest and direct way is to develop a converter between various basic data formats. Because there are not many popular data formats and the conversion rules are clear, this is a problem that can be solved as long as a certain amount of manpower is invested. However, semantic data mining clustering is more complicated, so this article conducts research on cloud computing-based data mining clustering algorithms.

Cluster analysis is to classify datasets based on similarity principles. Clustering is an algorithm in data mining, and it is a pivotal branch in data mining. Researchers have always regarded clustering algorithms as an important research direction, and clustering algorithms have been applied to many fields in major companies. Moreover, it plays an important role in text clustering, user group classification, biology, and finding outliers. The clustering algorithm can efficiently discover the internal laws of things, so it is an important step in data development. In addition, the clustering algorithm can divide the dataset into several classes according to certain rules and select the appropriate similarity measurement formula; thereby, the data belonging to the same class are similar to each other, but the data between the classes are not similar. The flow of the clustering algorithm is shown in Figure 1.

In machine learning, the clustering algorithm is an unsupervised learning algorithm, that is, there is no need to know the type of data before clustering. The purpose of clustering is to classify similar objects in the dataset into the same category. Generally, the evaluation of the clustering effect is determined by the corresponding clustering algorithm, sample approximate distance, and evaluation function. When the number of iterations reaches a certain value and the judgment function tends to infinity, the clustering achieves the best effect [24].

3.1. Sample Similarity Measurement. The following are several commonly used distance measurement methods:

(a) Euclidean distance: it is a distance calculation method used to find the distance between two points in the Euclidean space, that is, the straight-line distance between two points. It is usually suitable for the case where the standard of each vector is unified. The formula is

$$
d=\sum_{i=1}^{N} \sqrt{\left(X_{1 i}-X_{2 i}\right)^{2}}
$$

(b) Manhattan distance: it is to calculate the distance between city blocks (one intersection to the next intersection), also known as "taxi distance," and it can also calculate the sum of absolute wheelbases of coordinate axes between data points. The formula is

$$
\sum_{p}\left\|I_{1}^{p}-I_{2}^{p}\right\| .
$$

(c) Chebyshev distance: it detects the maximum value of the difference between the data points of two sample records in each coordinate axis. It can also be described as a one-dimensional attribute and is used to determine which cluster an object belongs to. The formula is

$$
d=\max \left(\left|x_{1}-x_{2}\right|,\left|y_{1}-y_{2}\right|\right) .
$$

(d) Cosine similarity: it can be described as the angle between two object attributes in space. The formula is

$$
d=\frac{\sum_{i=1}^{N} x_{i} y_{i}}{\sqrt{\sum x_{i}^{2}} \sqrt{\sum y_{i}^{2}}}
$$

This paper uses the Euclidean function to measure the similarity between data objects in the cluster. 


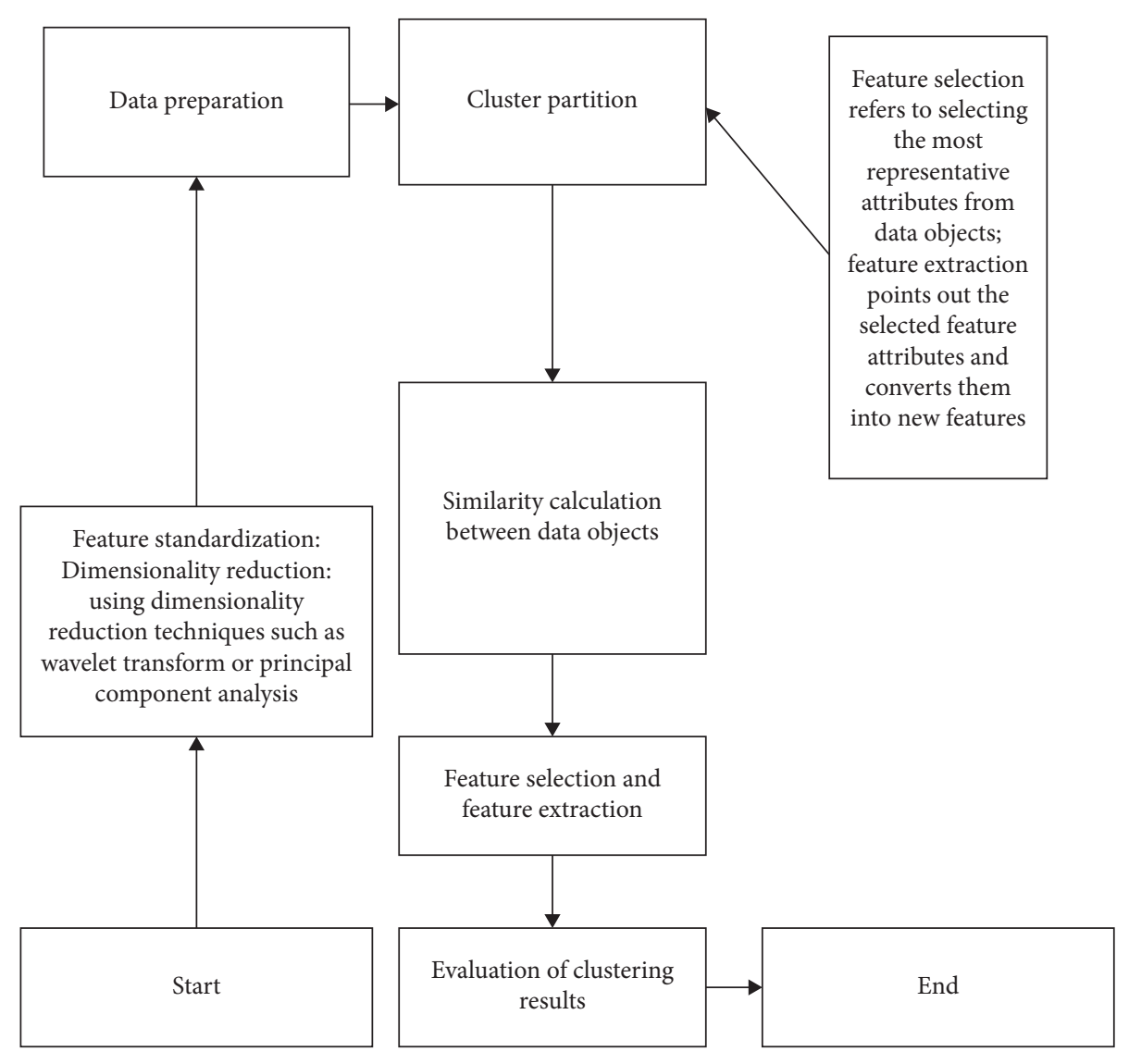

FIGURE 1: Clustering process.

\subsection{Evaluation of Clustering Effect}

(a) Davies-Bouldin index

$$
\mathrm{DB}=\frac{1}{n} \sum_{i=1}^{n} \max _{j \neq i}\left(\frac{\sigma_{i}+\sigma_{j}}{d\left(c_{i}, c_{j}\right)}\right) .
$$

In the above formula, $n$ represents the number of clusters, $c_{x}$ represents the centroid of the cluster $x, \sigma_{x}$ is the average distance from all data points in the $x$ cluster to the centroid $c_{x}$, and $d\left(c_{i}, c_{j}\right)$ represents the distance between the centroids $c_{i}$ and $c_{j}$. The smallest Davies-Bouldin index is considered to be the best algorithm based on this standard.

(b) Dunn index is used to evaluate clusters with close and easily distinguishable data. It is defined as the ratio between the smallest distance between clusters and the largest distance within clusters. The formula is calculated as follows:

$$
D=\frac{\min _{1 \leq i \leq j \leq n} d(i, j)}{\max _{1 \leq k \leq n} d^{\prime}(k)} .
$$

In the above formula, $d(i, j)$ represents the distance between the clusters $i$ and $j$ as well as $d^{\prime}(k)$ is the intracluster distance of cluster $k$. The intercluster distance $d(i, j)$ between two clusters can be any number of distance measures. Similarly, the distance $d^{\prime}(k)$ within the cluster can be measured in various ways, such as the maximum distance between any pair of elements in the cluster $k$. Since internal standards seek clusters with high intracluster similarity and low intercluster similarity, it is more desirable to generate an algorithm for clusters with a high Dunn index.

(c) Rand index: the Rand index calculates how similar the cluster is to the benchmark classification. In addition, the Rand index can also be as a measure of the percentage of correct decisions made by an algorithm. The formula is as follows:

$$
\mathrm{RI}=\frac{\mathrm{TP}+\mathrm{TN}}{\mathrm{TP}+\mathrm{FP}+\mathrm{FN}+\mathrm{TN}}
$$

$\mathrm{TP}$ represents the number of true examples, TN represents the number of true and negative examples, FP represents the number of false-positive examples, and FN represents the number of falsenegative examples. One problem with the Rand index is that false positives and false negatives are equally weighted. For some cluster applications, this may be an undesirable feature. 
(d) F-measure

$$
\begin{aligned}
P & =\frac{\mathrm{TP}}{\mathrm{TP}+\mathrm{FP}}, \\
R & =\frac{\mathrm{TP}}{\mathrm{TP}+\mathrm{FN}},
\end{aligned}
$$

where $P$ represents the precision rate and $R$ represents the recall rate. We can calculate the F-measure using the following formula:

$$
\mathrm{RI}=\frac{\mathrm{TP}+\mathrm{TN}}{\mathrm{TP}+\mathrm{FP}+\mathrm{FN}+\mathrm{TN}} .
$$

It should be noted that when $\beta=0, F_{0}=P$. In other words, at $\beta=0$, the recall rate has no effect on the F-measure.

According to the type of data and the purpose of clustering, researchers have pioneered the use of appropriate clustering algorithms to execute data of different dimensions and shapes so as to make the clustering results more accurate. Traditional clustering methods include categories: density-based clustering, probability distribution-based clustering, and hierarchical clustering.

The idea of the K-means Algorithms 1 and 2 is as follows: in the first step, $k$ values are randomly selected from the dataset as the cluster center; in the second step, the distance from each element to the center point is calculated, and these objects select the closest point to themselves as their own class; in the third step, the average value of all objects in all classes is calculated in order, and the average value is used as the center of the new class; in the fourth step, the average error and the $E$ value are calculated until it is less than the specified threshold; otherwise, the second step is followed and iteration is continued.

Randomly selecting $k$ data points as the initial center points of the clustering will make the clustering effect unstable. For datasets that are not convex, the randomness of the initial centers will usually cause a local optimal solution. Then, this function has many local optimal solutions, and only one of the many local optimal solutions is the global optimal solution. As shown in Figure 2, because the initial center points of the clustering are different, the path of the clustering objective function will follow four different paths $V_{a}, V_{b}, V_{c}, V_{d}$ to reach the local optimal solution. Among the four local optimal solutions, only one is the global optimal solution $\mathrm{C}$ is obtained through $V_{c}$-path iteration. Therefore, if the initial clustering center point selection is not appropriate, the K-means clustering algorithm will usually not find the global optimal point and stay at the local optimal point.

In addition, the number of clusters must be specified before K-means clustering. However, many datasets to be classified have unknown characteristics. If the number of dataset classes cannot be clearly indicated, it is necessary to perform multiple clustering tests on the number of different clusters $k$, and $k$ is selected with the best clustering effect as the number of clusters. This number is not taken randomly. Experiments show that this number must be obtained from $[2$, int $\sqrt{n}]$ so as to obtain the optimal solution for the evaluation clustering effect function. However, the K-means algorithm will be run multiple times in the process of finding the best $k$ value.

LDC is a novel clustering algorithm. The main ideas of the algorithm are as follows: (1) calculating the local density values of all data and arranging them in descending order, (2) selecting the value with high local density as the clustering center point, and (3) clustering the noncentral point data to the nearest class. Several parameters of LDC are defined as follows.

Definition 1. Local density

$$
\begin{aligned}
\rho_{i} & =\sum_{i} \chi\left(d_{i j}-d_{c}\right) . \\
\chi(i) & =\left\{\begin{array}{ll}
1 & i<0 \\
0 & i \geq 0
\end{array},\right.
\end{aligned}
$$

where $d_{i j}$ represents the distance between data points $i$ and $j$ and $d_{c}$ is the cutoff distance and is an adjustable parameter. $\rho_{i}$ represents the number of data points in the range with $i$ as the center and $d_{c}$ as the radius. The LDC algorithm is more sensitive to the value of $\rho_{i}$, so the value of the cutoff distance $d_{c}$ has a great influence on the clustering effect.

Definition 2. High-density minimum distance

$$
\delta_{i}=\min _{j: \rho_{j}>\rho_{i}} d_{i j}
$$

where $d_{i j}$ represents the distance between data points $i$ and $j$ and the high-density minimum distance $\delta_{i}$ represents the distance from data point $i$ to the nearest point $j$ among all the points with a local density greater than data point $i$. For the point with the highest local density, it is defined as

$$
\delta_{i}=\max \left(d_{i j}\right)
$$

Below, we have provided an example to analyze the LDC algorithm in detail.

(1) Decision diagram: LDC is a two-dimensional algorithm. The algorithm performs clustering according to Figure 3. Figure 3(a) shows the distribution diagram of the data, and Figure 3(b) shows the decision diagram of the data in Figure 3(a). The labels of the sample points are arranged in descending order according to the local density. Different colors represent different clusters, and black dots are noise dots.

The judgment of this clustering center is mainly through the human eyes. From the decision diagram, it is easy to find that point 1 and point 10 are obviously different from other data points, and they have a relatively large local density $\rho$ and a relatively large high-density minimum distance $\delta$. Therefore, 
Input: $k$ value and dataset $D\left(D_{1}, D_{2}, D_{3}, \ldots, D_{i}, \ldots, D_{n}\right)$.

Output: clustering results.

(a) This algorithm randomly selects $k$ data in the dataset $D$ as the initial cluster center point.

(b) This algorithm finds distance from all remaining data points to the $k$ cluster center points and selects the closest point to itself as its cluster center point.

(c) The algorithm calculates the center points of all classes in turn and uses these center points as the new cluster centers.

(d) The algorithm iterates steps $\mathrm{b}$ and $\mathrm{c}$ repeatedly until the error average and $E=\sum_{i=1}^{k} \sum_{x}\left|x-\bar{x}_{i}\right|^{2}$ value are less than the specified threshold. The algorithm ends.

Algorithm 1: K-means algorithm [16].

Input: intercept $d_{c}$.

Output: clustering results.

(a) The algorithm finds the distance between all data.

(b) The algorithm calculates the local density $\rho_{i}$ of all data points.

(c) The algorithm calculates the high-density minimum distance $\delta_{i}$ of all data.

(d) The algorithm finds the cluster center point in the decision graph.

(e) The algorithm clusters the remaining points to the nearest center point.

(f) The algorithm removes noise points.

Algorithm 2: LDC algorithm [18].

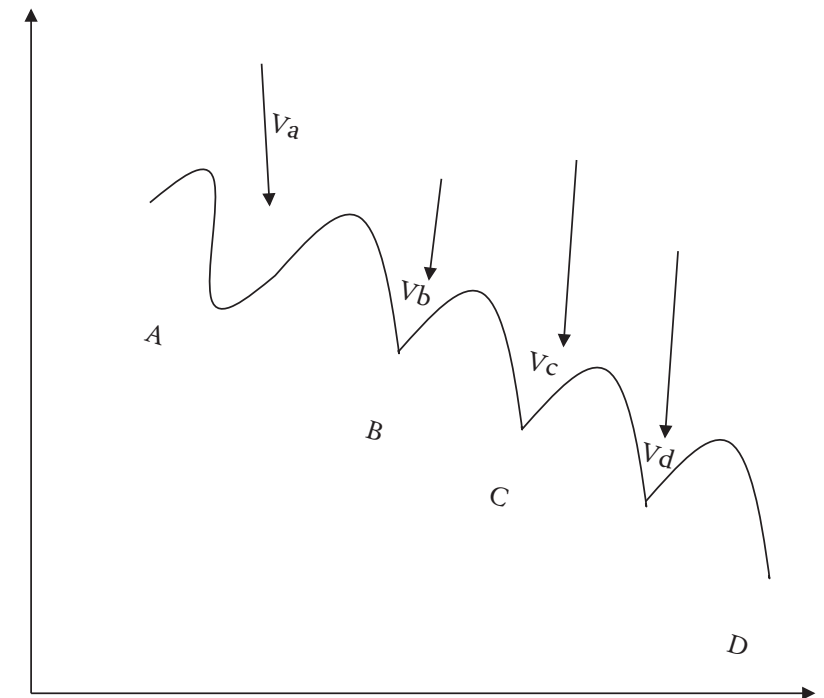

Figure 2: The space state of the error sum of squares criterion function.

they are used as the center of the cluster when clustering. Although points 26, 27, and 28 have larger high-density minimum distance $\delta$, the local density $\rho$ is smaller. Therefore, they are deleted as noise points.

(2) Classification of data points: after determining the cluster center point in the decision diagram, the remaining data is clustered to the center point closest to itself. The algorithm is simple and clear and has low complexity and no iteration. After the algorithm establishes the center point, clustering is performed directly.
(3) Noise filtering: noise points will affect the clustering effect. The algorithm removes noise points by a boundary density. If the local density $\rho$ of the data satisfies $\rho_{i} \leq \mu(\rho)-2 \sigma(\rho)$, it is defined as a noise point and deleted from the dataset.

Aiming at the problem that the clustering results cannot reach the global optimum caused by the random selection of the initial center point of $\mathrm{K}$-means and the influence of noise points on the clustering, this paper proposes the LDCKmeans algorithm and conducts clustering experiments to verify its effectiveness. The K-means algorithm also has the following shortcomings: the quality of clustering is sensitive to the random selection of initial points, the selection of $k$ value should be specified, and clustering is also more sensitive to existing noise points. Next, this article analyzes these shortcomings in detail, then makes corresponding optimizations, and finally parallelizes the optimized algorithms to improve the ability to process large-scale data.

The definition of some parameters of the AK-means algorithm is as follows:

Definition 3. Intercluster dispersion degree

$$
\text { Disp }=\frac{\sum_{i=1}^{k} \operatorname{Disp}_{i}}{k}
$$

Disp represents the intercluster dispersion degree, $\operatorname{disp}_{i}$ means the position of the i-th cluster center point, and $k$ means the number of clusters.

Definition 4. Intracluster aggregation degree

$$
\text { Aggr }=\frac{\sum_{i=1}^{k} \operatorname{Aggr}_{i}}{k}
$$




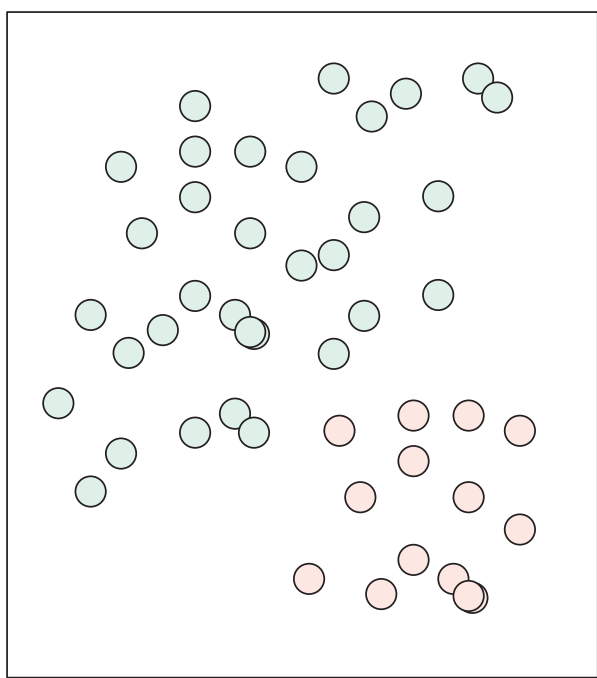

(a)

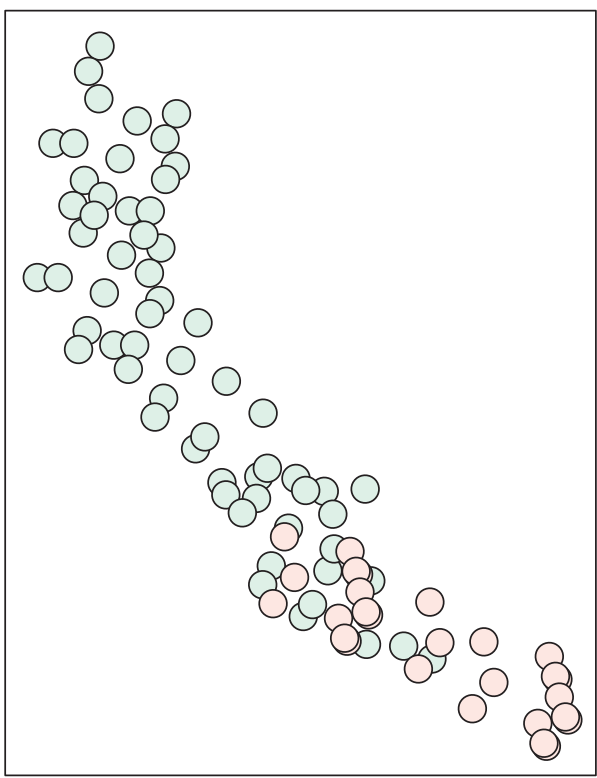

(b)

Figure 3: Data distribution diagram and decision diagram. (a) Data point distribution diagram. (b) Decision diagram.

Aggr represents the intracluster aggregation degree, $k$ represents the number of clusters, and Aggr $_{i}$ represents the average distance from all elements in the $\mathrm{i}$-th cluster to the center.

Definition 5. Clustering evaluation value

$$
E=\frac{\operatorname{Aggr}_{k}-\text { Aggr }_{k-1}}{\operatorname{Disp}_{k}-\text { Disp }_{k-1}},
$$

whereDisp $_{k}$ means the $\mathrm{k}$-th intercluster dispersion degree, Disp $_{k-1}$ means the $\mathrm{k}$-th intercluster dispersion degree, $\mathrm{Aggr}_{k}$ means the k-th intracluster aggregation degree, Aggr $_{k-1}$ means the $k-1$-th intracluster aggregation degree, and $E$ is the cluster evaluation value.

To determine the effectiveness of AK-means, we obtain data from the UCI database and choose Iris and Wine as the experimental dataset. Their data volumes are 150 and 178 , respectively, the data attributes are 4 and 3, respectively, and the data types are all 3.

To show the differences of the evaluation values $E$ as well as $\log _{2}^{E}-\log _{2}^{E_{0}}$ in the clustering process of Iris and Wine, a line graph is drawn as shown in Figure 4.

In Figure 4, while the value of $k$ is larger than 2, the value of $E$ has a small alteration, and the value of $\log _{2}^{E}-\log _{2}^{E_{0}}$ is less than 1 , which indicates that the algorithm can continue to iterate as well as the combination is reasonable. While the value of $k$ alters from 3 to 2, the value of $E$ suddenly increases, and the value of $\log _{2}^{E}-\log _{2}^{E_{0}}$ is larger than 1 . This indicates that the merging is unreasonable, so the number of clusters in both datasets is 3 . In addition, these experimental results are lined in the real number 3 of the two datasets. This demonstrates the effectiveness of AKmeans.

\section{Construction of the Intelligent Network Office System Based on Cloud Computing and Machine Learning}

The overall system architecture design adopts a serviceoriented (SOA) construction idea, that is, using a mature and stable multilayer architecture, adopting an overall plan, and adopting a step-by-step implementation. The overall architecture is shown in Figure 5.

Based on the abovementioned overall architecture, the smart office application system platform is designed according to the following steps: (1) the system adopts original intelligent desktop technology to create a new user experience; (2) the system uses cloud service technology to build smart applications of "desktop as a service"; (3) the system relies on the cloud computing platform to realize application services. Through multiple types of interactive terminals (computers, notebooks, smart phones, and tablet computers), a multiterminal application interconnected and shared smart service is formed.

The system technical architecture is designed according to the SOA design guiding ideology and multilayer architecture. The technical architecture is shown in Figure 6. Using Java mature SSH framework and development technology, it is developed according to the technical requirements of high cohesion, low coupling, pluggability, and componentization to realize the requirements of development of overall applications, high reusability, and flexible expansion and integration.

Private clouds are built for a customer to use alone to provide the most effective control over data, security, and service quality. The unit owns the infrastructure as well as can control the applications deployed on this infrastructure. Private clouds can be deployed in the firewall of university data centers, or they can be deployed in a secure hosting 


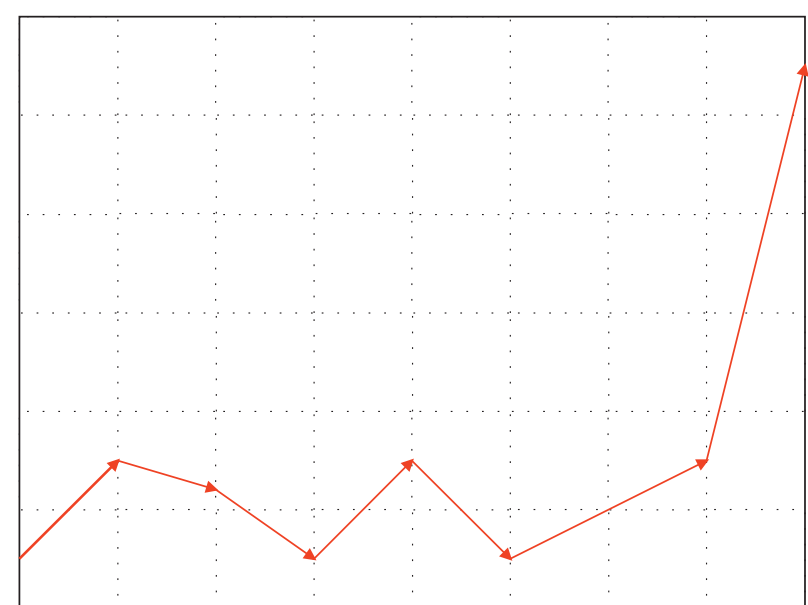

(a)

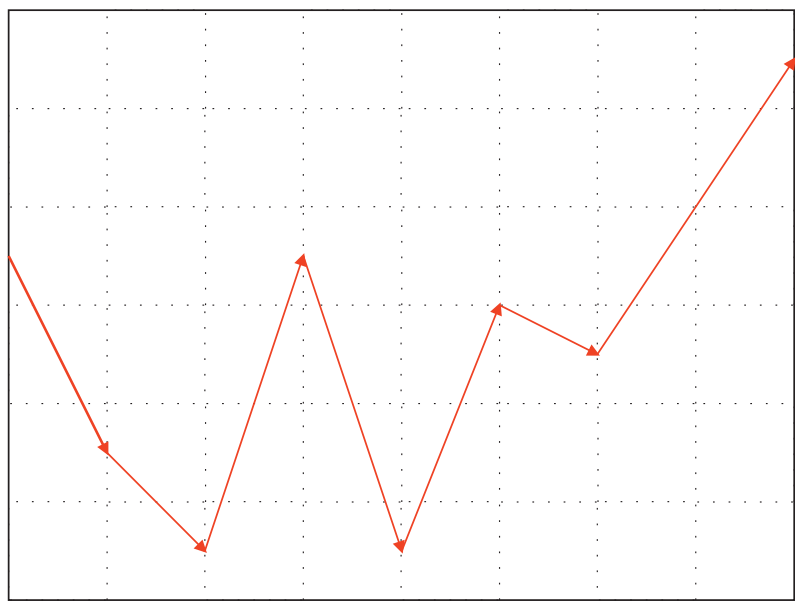

(c)

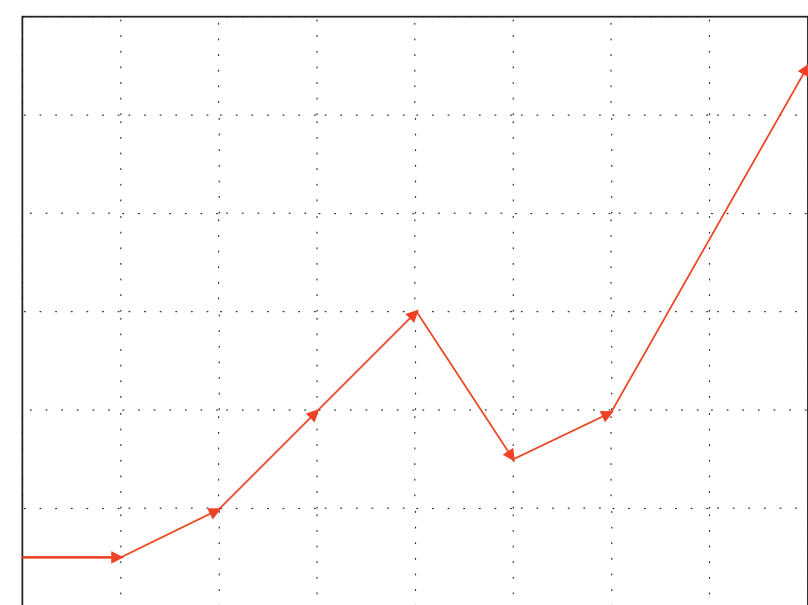

(b)

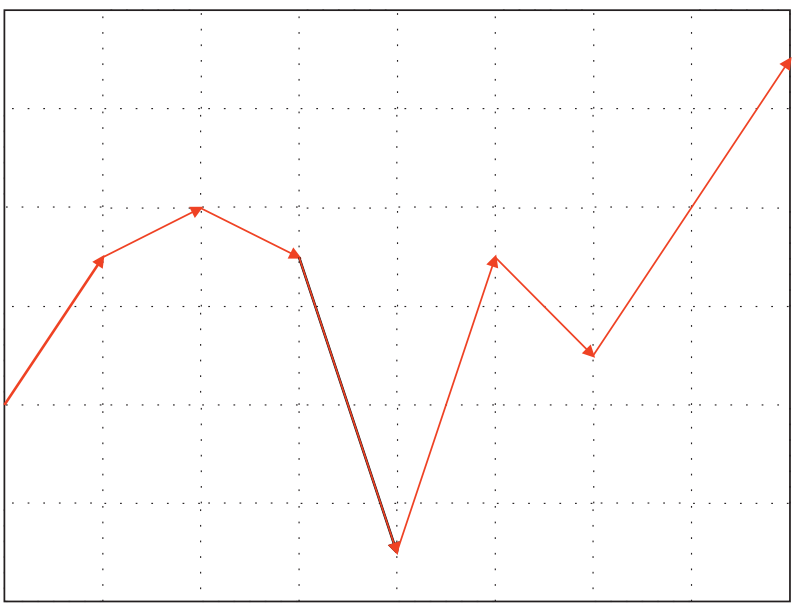

(d)

Figure 4: Line chart of the evaluation values $E$ and $\log _{2}^{E}-\log _{2}^{E_{0}}$ of Iris and Wine E. (a) The change of Iris' evaluation value E. (b) The change of Wine's judgment value $E$. (c) The change of Iris' evaluation value $\log _{2}^{E}-\log _{2}^{E_{0}}$. (d) The change of Wine's judgment value $\log _{2}^{E}-\log _{2}^{E_{0}}$.

location. In this study, the private cloud is deployed in the university firewall, and the design of basic cloud services must comply with the following principles: (1) It needs to focus on the first phase and consider future scalability. (2) It needs to deploy cloud architecture flexibly to facilitate management and maintenance. (3) It can be observed at any time. (4) Ensure data security. (5) All processes are automated. Figure 7 is a specific planning model for the basic cloud service design.

In the planning model, the logical structure of the corresponding basic cloud service design is shown in Figure 8.

\section{Performance Evaluation of Intelligent Network Office System Based on Cloud Computing and Machine Learning}

This article combines cloud computing and machine learning technology to build an intelligent networked office system and combines demand analysis to evaluate the performance of the intelligent networked office system. The office system requires a large amount of data fusion work, and it needs to have a strong humanization and convenient network office effect, so this article conducts system performance verification with the support of cloud computing and machine learning technology. First, this article evaluates the data fusion effect of the system, and the results are illustrated in Table 1 and Figure 9.

Compared with the literature [13], the method proposed in this article has more advantages in data fusion. The data fusion in this article is based on the first line of cloud computing, so the speed of data fusion is more advantageous.

From the analysis of Figure 9 and Table 2, we can see that the system constructed in this paper performs well in terms of data fusion and can basically meet the needs of daily smart office. On this basis, this paper conducts a satisfaction survey on the office system constructed in this paper, and the operating effect of the system can be reflected through the satisfaction degree. The statistical results of satisfaction are illustrated in Table 2 and Figure 10. 


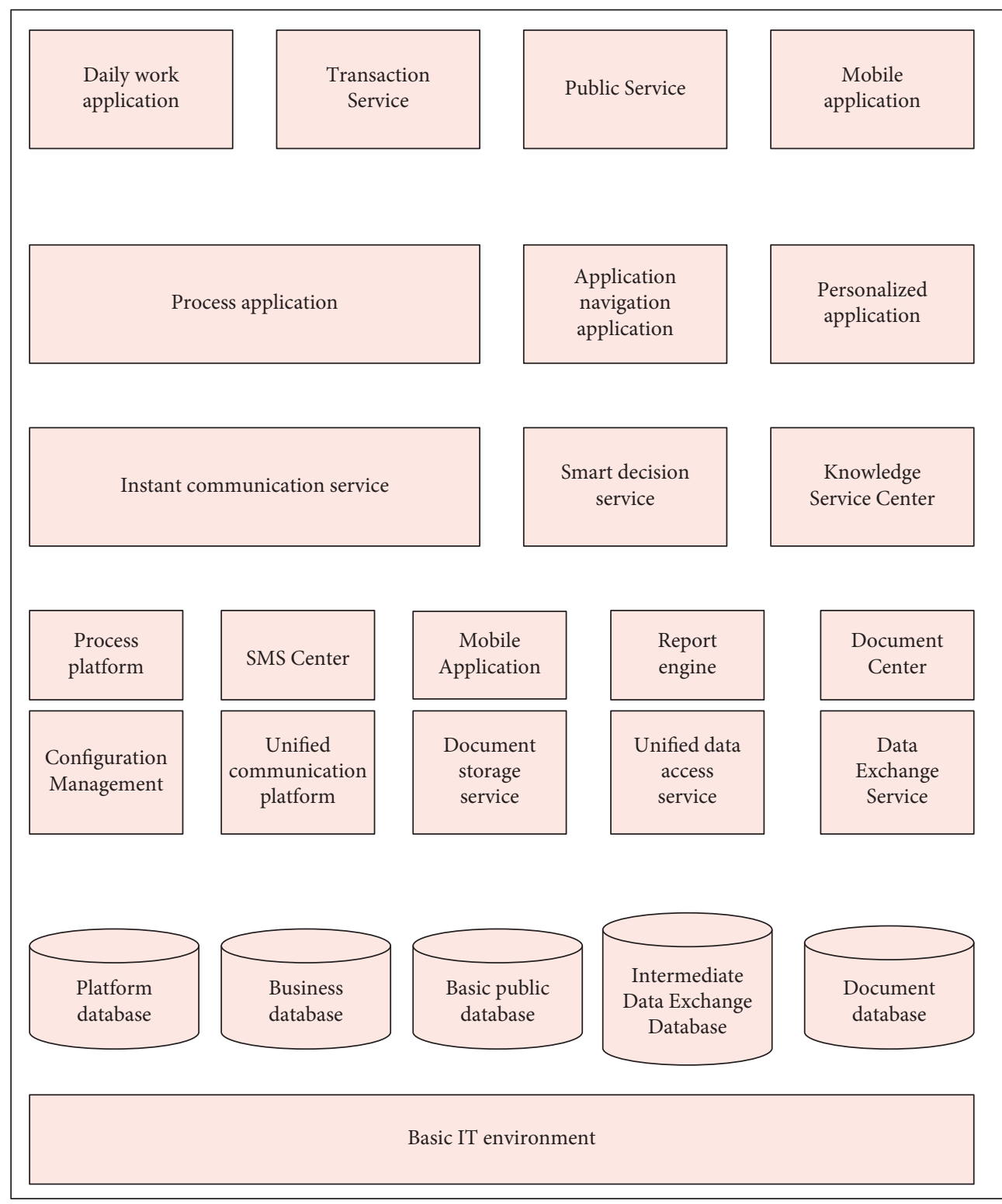

FIGURE 5: Overall architecture. 


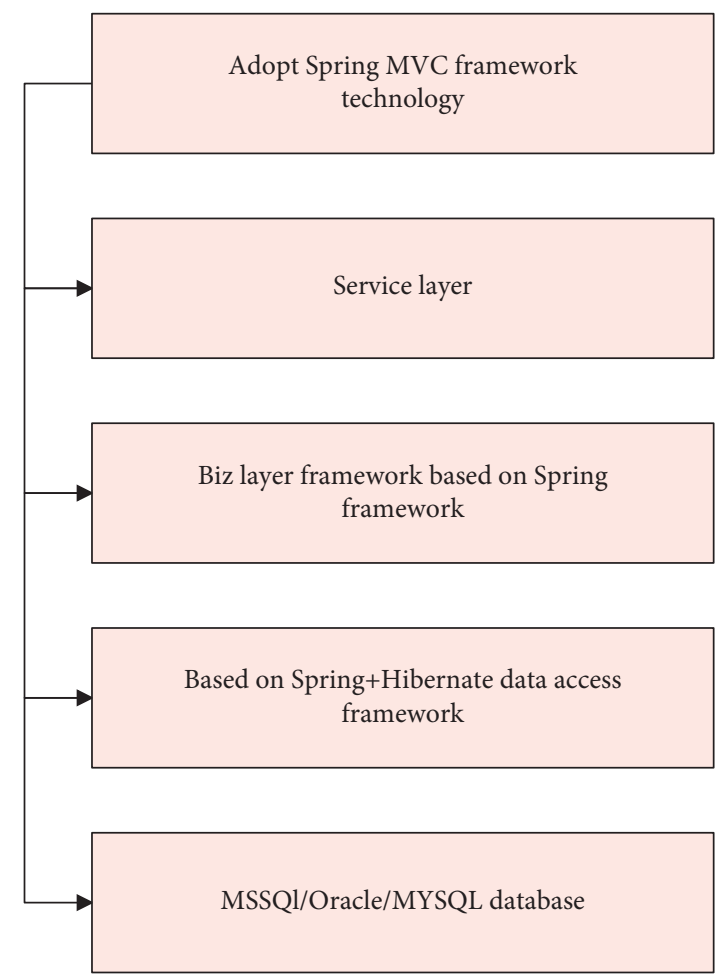

FIgure 6: Technical architecture diagram.

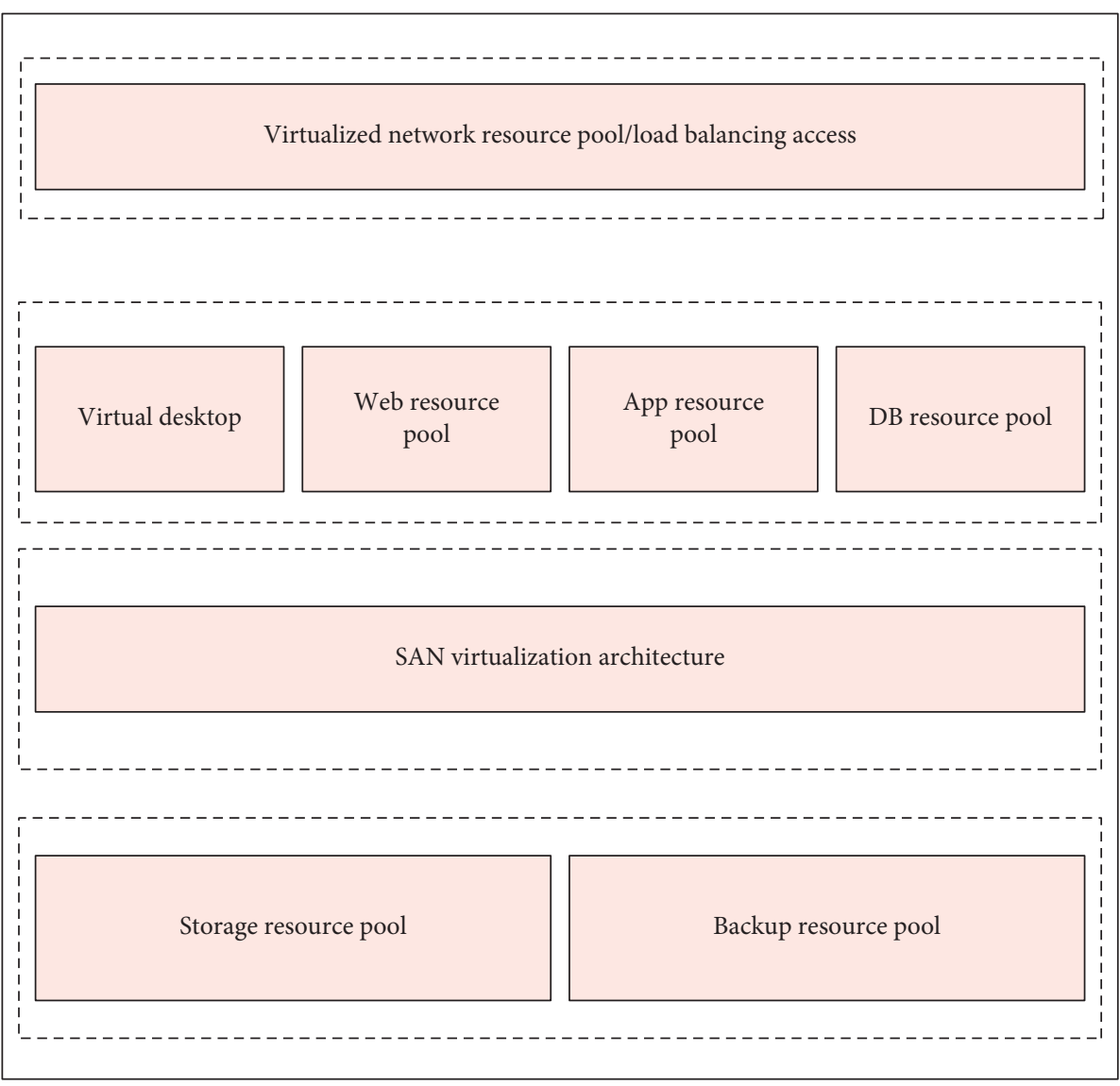

FIgURE 7: Basic cloud service design planning model. 


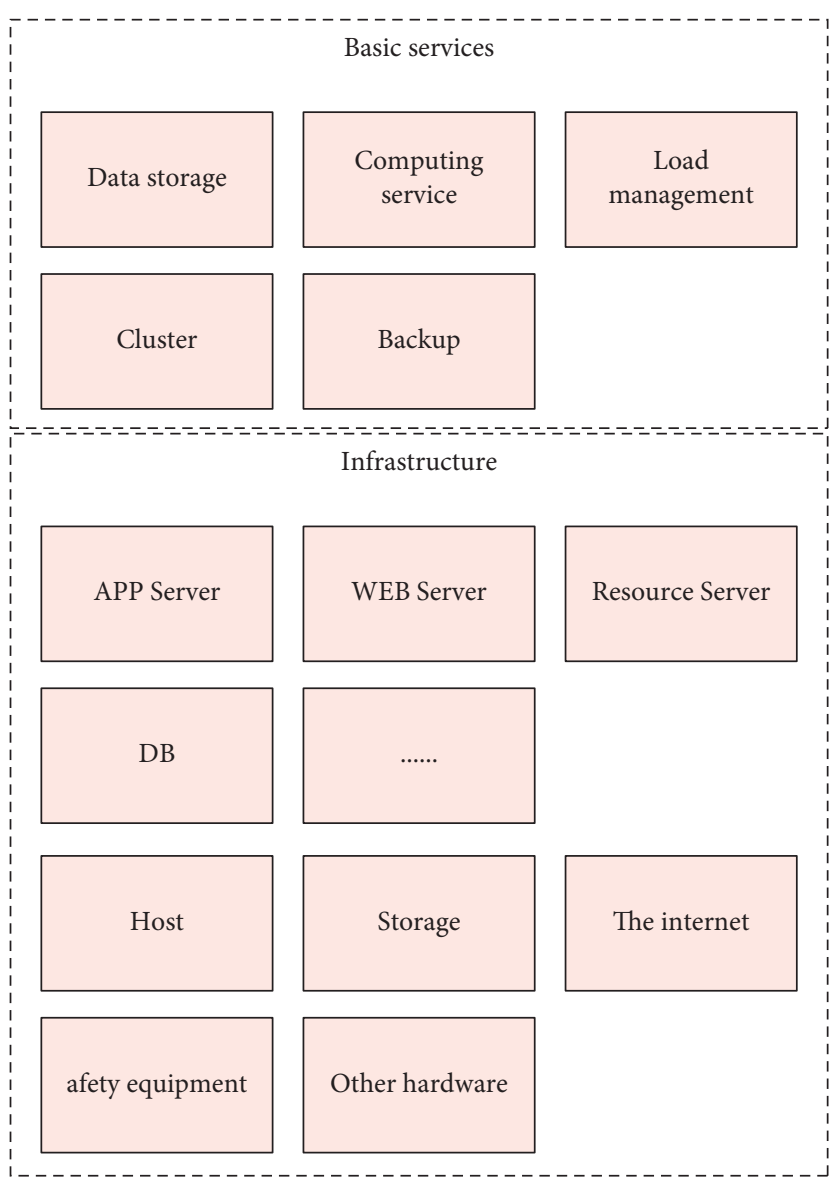

FIGURE 8: Logical structure of basic cloud service design.

TABLe 1: Statistical table of system data fusion effect.

\begin{tabular}{|c|c|c|c|c|c|}
\hline Num & Data fusion & Num & Data fusion & Num & Data fusion \\
\hline 1 & 96.5 & 27 & 96.7 & 53 & 96.4 \\
\hline 2 & 97.8 & 28 & 95.7 & 54 & 98.2 \\
\hline 3 & 98.0 & 29 & 95.9 & 55 & 95.5 \\
\hline 4 & 97.8 & 30 & 97.5 & 56 & 95.0 \\
\hline 5 & 95.5 & 31 & 97.1 & 57 & 96.1 \\
\hline 6 & 95.2 & 32 & 96.3 & 58 & 96.1 \\
\hline 7 & 98.9 & 33 & 97.6 & 59 & 98.7 \\
\hline 8 & 97.1 & 34 & 98.9 & 60 & 95.9 \\
\hline 9 & 95.8 & 35 & 98.8 & 61 & 97.5 \\
\hline 10 & 96.4 & 36 & 97.0 & 62 & 97.4 \\
\hline 11 & 95.2 & 37 & 96.4 & 63 & 98.3 \\
\hline 12 & 98.5 & 38 & 95.2 & 64 & 95.4 \\
\hline 13 & 95.5 & 39 & 98.9 & 65 & 96.6 \\
\hline 14 & 95.1 & 40 & 98.2 & 66 & 96.6 \\
\hline 15 & 95.7 & 41 & 97.8 & 67 & 95.8 \\
\hline 16 & 97.3 & 42 & 95.7 & 68 & 97.5 \\
\hline 17 & 95.8 & 43 & 95.7 & 69 & 95.6 \\
\hline 18 & 95.2 & 44 & 95.9 & 70 & 96.8 \\
\hline 19 & 96.6 & 45 & 97.4 & 71 & 99.0 \\
\hline 20 & 96.2 & 46 & 97.7 & 72 & 98.4 \\
\hline 21 & 96.3 & 47 & 95.4 & 73 & 98.1 \\
\hline 22 & 95.6 & 48 & 96.4 & 74 & 95.3 \\
\hline 23 & 97.6 & 49 & 97.0 & 75 & 95.9 \\
\hline 24 & 96.8 & 50 & 98.6 & 76 & 97.7 \\
\hline 25 & 95.5 & 51 & 98.7 & 77 & 98.7 \\
\hline 26 & 99.0 & 52 & 96.5 & 78 & 96.9 \\
\hline
\end{tabular}




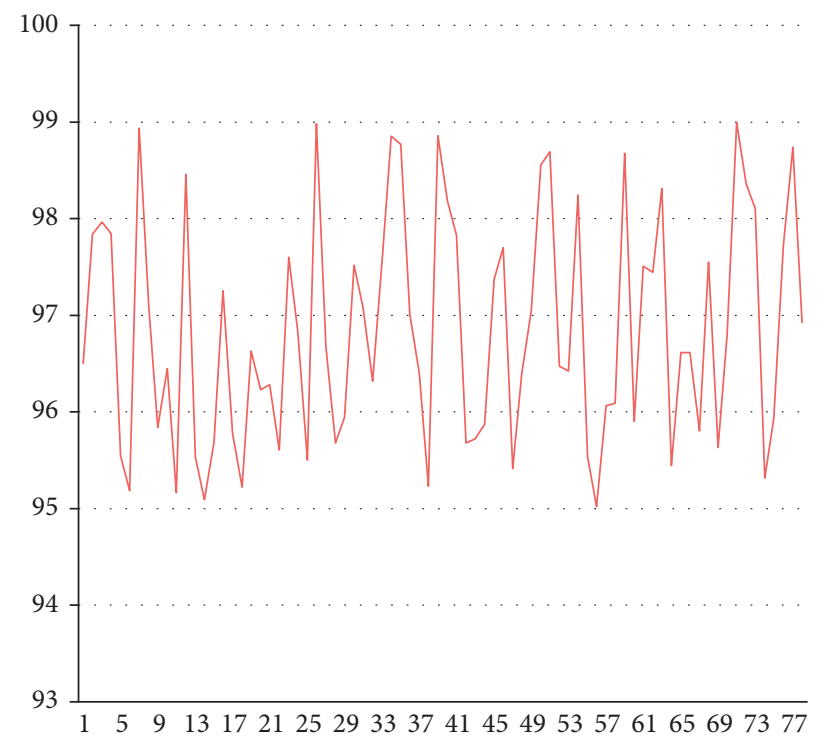

FIGURE 9: Statistical diagram of system data fusion effect.

TABLE 2: Statistical table of the results of the satisfaction survey of the intelligent office system.

\begin{tabular}{|c|c|c|c|c|c|}
\hline Num & Satisfaction & Num & Satisfaction & Num & Satisfaction \\
\hline 1 & 91.5 & 27 & 91.8 & 53 & 94.6 \\
\hline 2 & 82.2 & 28 & 90.6 & 54 & 87.8 \\
\hline 3 & 85.5 & 29 & 97.2 & 55 & 94.2 \\
\hline 4 & 92.8 & 30 & 95.5 & 56 & 84.3 \\
\hline 5 & 84.9 & 31 & 90.1 & 57 & 87.9 \\
\hline 6 & 94.7 & 32 & 93.2 & 58 & 96.9 \\
\hline 7 & 89.3 & 33 & 93.3 & 59 & 83.4 \\
\hline 8 & 86.7 & 34 & 86.0 & 60 & 85.8 \\
\hline 9 & 85.8 & 35 & 85.5 & 61 & 87.4 \\
\hline 10 & 86.3 & 36 & 89.5 & 62 & 87.1 \\
\hline 11 & 94.4 & 37 & 85.9 & 63 & 88.7 \\
\hline 12 & 84.7 & 38 & 90.3 & 64 & 93.4 \\
\hline 13 & 89.6 & 39 & 83.1 & 65 & 83.0 \\
\hline 14 & 97.0 & 40 & 83.0 & 66 & 83.7 \\
\hline 15 & 89.3 & 41 & 85.7 & 67 & 97.2 \\
\hline 16 & 88.4 & 42 & 97.9 & 68 & 92.5 \\
\hline 17 & 93.3 & 43 & 93.7 & 69 & 89.9 \\
\hline 18 & 94.5 & 44 & 82.9 & 70 & 91.2 \\
\hline 19 & 96.0 & 45 & 85.6 & 71 & 94.8 \\
\hline 20 & 91.3 & 46 & 83.1 & 72 & 85.8 \\
\hline 21 & 94.4 & 47 & 90.8 & 73 & 90.9 \\
\hline 22 & 96.9 & 48 & 93.1 & 74 & 93.4 \\
\hline 23 & 84.8 & 49 & 87.0 & 75 & 96.6 \\
\hline 24 & 88.2 & 50 & 92.0 & 76 & 83.0 \\
\hline 25 & 92.2 & 51 & 87.0 & 77 & 85.5 \\
\hline 26 & 84.0 & 52 & 95.6 & 78 & 87.7 \\
\hline
\end{tabular}

Compared with traditional paperless office methods and existing intelligent office methods, the method in this article has a certain improvement in office intelligence and office efficiency.
From the above statistical results, we can see that the intelligent office system is highly satisfactory. It can be seen that the effect of the intelligent office system constructed in this article meets the expected requirements. 


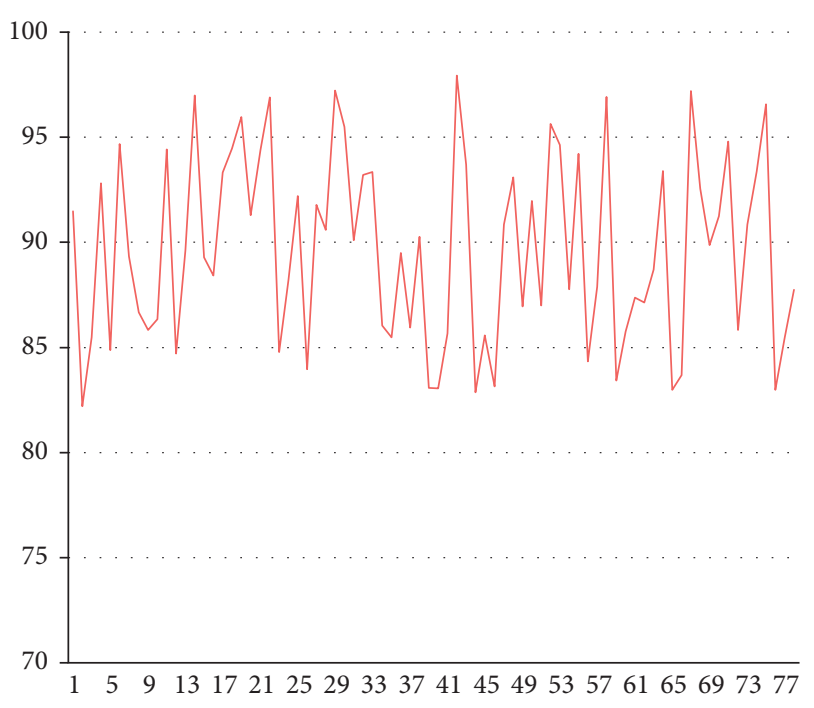

Figure 10: Statistical diagram of the results of the satisfaction survey of the intelligent office system.

\section{Conclusion}

The traditional office automation system focuses on basic office transaction processing functions, and its ability to support process management is weak. Moreover, the part of the workflow is realized manually, so the work intensity is high and the work efficiency is low, and end-to-end closedloop management cannot be realized. Aiming at the problem of insufficient traditional office management and process management capabilities, this article combines cloud computing and machine learning to construct an intelligent network office system. At the same time, this paper uses the combination of IoT data mining technology and cloud computing framework to realize large-scale IoT data processing and proposes functional and nonfunctional requirements for an office automation management system based on an intelligent workflow. After that, according to the functional and nonfunctional requirements of the office automation management system, this article carries out the overall design of the office automation management system based on an intelligent workflow. Finally, this article combines the actual needs to evaluate the performance of the intelligent network office system. From the actual research situation, the intelligent network office system constructed in this paper has certain effects.

\section{Data Availability}

The labeled dataset used to support the findings of this study is available from the corresponding author upon request.

\section{Conflicts of Interest}

The authors declare no conflicts of interest.

\section{Acknowledgments}

This study was sponsored by Taiyuan University.

\section{References}

[1] W. Tushar, N. Wijerathne, W.-T. Li et al., "Internet of things for green building management: disruptive innovations through low-cost sensor technology and artificial intelligence," IEEE Signal Processing Magazine, vol. 35, no. 5, pp. 100-110, 2018.

[2] J. H. Abawajy and M. M. Hassan, "Federated internet of things and cloud computing pervasive patient health monitoring system," IEEE Communications Magazine, vol. 55, no. 1, pp. 48-53, 2017.

[3] D. He, R. Ye, S. Chan, M. Guizani, and Y. Xu, "Privacy in the internet of things for smart healthcare," IEEE Communications Magazine, vol. 56, no. 4, pp. 38-44, 2018.

[4] P. P. Ray, "Internet of things for smart agriculture: technologies, practices and future direction," Journal of Ambient Intelligence and Smart Environments, vol. 9, no. 4, pp. 395420, 2017.

[5] Y. A. Qadri, A. Nauman, Y. B. Zikria, A. V. Vasilakos, and S. W. Kim, "The future of healthcare internet of things: a survey of emerging technologies," IEEE Communications Surveys \& Tutorials, vol. 22, no. 2, pp. 1121-1167, 2020.

[6] B. H. Dobkin, "A rehabilitation-internet-of-things in the home to augment motor skills and exercise training," $\mathrm{Neu}$ rorehabilitation and Neural Repair, vol. 31, no. 3, pp. 217-227, 2017.

[7] J. Yao and N. Ansari, "Caching in energy harvesting aided Internet of Things: a game-theoretic approach[J]," IEEE Internet of Things Journal, vol. 6, no. 2, pp. 3194-3201, 2018.

[8] J. E. Siegel, S. Kumar, and S. E. Sarma, "The future internet of things: secure, efficient, and model-based," IEEE Internet of Things Journal, vol. 5, no. 4, pp. 2386-2398, 2017.

[9] M. A. Abd-Elmagid, N. Pappas, and H. S. Dhillon, "On the role of age of information in the internet of things," IEEE Communications Magazine, vol. 57, no. 12, pp. 72-77, 2019.

[10] A. Sheth, U. Jaimini, and H. Y. Yip, "How will the internet of things enable augmented personalized health?" IEEE Intelligent Systems, vol. 33, no. 1, pp. 89-97, 2018.

[11] G. J. Joyia, R. M. Liaqat, and A. Farooq, "Internet of Medical Things (IOMT): applications, benefits and future challenges in healthcare domain," Journal of Communication, vol. 12, no. 4, pp. 240-247, 2017.

[12] N. Kshetri, "The evolution of the internet of things industry and market in China: an interplay of institutions, demands and supply," Telecommunications Policy, vol. 41, no. 1, pp. 49-67, 2017.

[13] S. Siboni, V. Sachidananda, Y. Meidan et al., "Security testbed for internet-of-things devices," IEEE Transactions on Reliability, vol. 68, no. 1, pp. 23-44, 2019.

[14] Y. Yang, M. Zhong, H. Yao, F. Yu, X. Fu, and O. Postolache, "Internet of things for smart ports: technologies and challenges," IEEE Instrumentation and Measurement Magazine, vol. 21, no. 1, pp. 34-43, 2018.

[15] Z. Li, Y. Liu, and A. Liu, "Minimizing convergecast time and energy consumption in green internet of things," IEEE Transactions on Emerging Topics in Computing, vol. 8, no. 3, pp. 797-813, 2018.

[16] P. P. Ray, "A survey on internet of things architectures," Journal of King Saud University-Computer and Information Sciences, vol. 30, no. 3, pp. 291-319, 2018.

[17] M. Mayer and A. J. Baeumner, "A megatrend challenging analytical chemistry: biosensor and chemosensor concepts ready for the internet of things," Chemical Reviews, vol. 119, no. 13, pp. 7996-8027, 2019. 
[18] M. Saez, F. P. Maturana, K. Barton, and D. M. Tilbury, "Realtime manufacturing machine and system performance monitoring using internet of things," IEEE Transactions on Automation Science and Engineering, vol. 15, no. 4, pp. 1735-1748, 2018.

[19] V. Jagadeeswari, V. Subramaniyaswamy, and R. Logesh, "A study on medical Internet of Things and Big Data in personalized healthcare system," Health Information Science and Systems, vol. 6, no. 1, pp. 1-20, 2018.

[20] S. Smys, A. Basar, and H. Wang, "Hybrid intrusion detection system for internet of things (IoT)," Journal of ISMAC, vol. 2, no. 04, pp. 190-199, 2020.

[21] I. Butun, P. Österberg, and H. Song, "Security of the internet of things: vulnerabilities, attacks, and countermeasures," IEEE Communications Surveys \& Tutorials, vol. 22, no. 1, pp. 616-644, 2019.

[22] T. Qiu, N. Chen, K. Li, M. Atiquzzaman, and W. Zhao, "How can heterogeneous internet of things build our future: a survey," IEEE Communications Surveys \& Tutorials, vol. 20, no. 3, pp. 2011-2027, 2018.

[23] A. Heiskanen, "The technology of trust: how the internet of things and blockchain could usher in a new era of construction productivity," Construction Research and Innovation, vol. 8, no. 2, pp. 66-70, 2017.

[24] M. Wolf and D. Serpanos, "Safety and security in cyberphysical systems and internet-of-things systems," Proceedings of the IEEE, vol. 106, no. 1, pp. 9-20, 2017. 